\title{
Spillovers de Volatilidades Cambiais e de Mercados Financeiros Internacionais no Mercado Acionário Brasileiro
}

\author{
(Spillovers of Foreign Exchange Volatility and International \\ Financial Markets in the Brazilian Stock Market)
}

\author{
Wendy Sidon Meira de Oliveira* \\ André Nunes Maranhão**
}

\begin{abstract}
Resumo
Apresentamos nesse estudo os resultados de transbordamento de volatilidades no mercado acionário brasileiro, medido por meio de correlações condicionais. Utilizando modelos GARCH multivariados foram estimadas as correlações condicionais em 3 diferentes modelos combinando o índice Ibovespa com um dos três tipos de choques cambiais e um choque de mercados financeiros internacionais. A existência e direção dos transbordamentos de volatilidade de choques cambias, choques de mercados financeiros internacionais e o índice Ibovespa foram testadas por meio de testes de causalidade de Granger de segunda ordem. Os resultados comprovam a existência de transbordamentos de choques cambiais e de mercados financeiros para o índice Ibovespa, sendo que essas correlações apresentam dinâmica temporal, com transbordamentos sempre na direção dos choques para o índice Ibovespa.
\end{abstract}

Palavras-chave: GARCH Multivariados, Causalidade de Granger de Segunda Ordem, Spillover de volatilidade, Choques Cambiais, Choques de Mercados Financeiros Internacionais

Códigos JEL: C58, G15, G17.

Submetido em 20 de julho de 2016. Reformulado em 18 de maio de 2018. Aceito em 21 de maio de 2018. Publicado on-line em 20 de junho de 2018. O artigo foi avaliado segundo o processo de duplo anonimato além de ser avaliado pelo editor. Editor responsável: Márcio Laurini.

*Universidade de Brasília e Banco do Brasil S/A, Brasília, DF, Brasil. Email: wendysidon@gmail.com

**Universidade de Brasília e Banco do Brasil S/A, Brasília, DF, Brasil. Email: andrenmaranhao@gmail.com

\footnotetext{
Rev. Bras. Finanças (Online), Rio de Janeiro, 15, No. 4, Oct. 2017, pp. 569-603 ISSN 1679-0731, ISSN online 1984-5146

(C)2017 Sociedade Brasileira de Finanças, under a Creative Commons Attribution 3.0 license http://creativecommons.org/licenses/by/3.0
} 


\begin{abstract}
We present in this study the results of volatility spillover in the Brazilian stock market, measured by conditional correlations. Using GARCH multivariate conditional correlations were estimated at 3 different models combining the Ibovespa index of the three types of exchange rate shocks and a shock of international financial markets. The existence and direction of spillovers of volatility of forward exchange shocks, international financial market shocks and the Ibovespa were tested by Granger causality test of second order. The results show the existence of spillovers from exchange rate shocks and financial markets for the Ibovespa index, and these correlations have temporal dynamics, with spillovers always in the direction of the shocks to the Ibovespa index.
\end{abstract}

Keywords: Multivariate GARCH, Causality Granger Second Order, Spillover volatility, Shocks Exchange, Shocks International Financial Markets.

\title{
1. Introdução
}

Desde da crise financeira que deflagrou em 2007 nos EUA, a literatura sobre spillover de volatilidade e contágio financeiro cresceu de forma significativa, com desenvolvimento e aplicação de novas ferramentas para o estudo (IMF (2016), Diebold and Yilmaz (2015)). Dornbusch and Fischer (1980) assumem que variações da taxa de câmbio afetam a competitividade das empresas, influenciando o retorno das ações. Por outro lado, outras teorias, como Branson (1983), Frankel and Froot (1985) apresentam um contra ponto. A relação entre preços de ações e taxas de câmbio em seus estudos pioneiros importaram-se com as variações em nível. Esses estudos evoluíram para tratar a volatilidade dos preços de ações e da taxa de câmbio, essa investigação evoluiu para o que a litertura chama de transbordamentos (spillover) de volatilidades. Uma questão relevante nesses estudos é identificar a direção desse transbordamento: do câmbio para ações ou vice-versa. Nesse contexto, muitos modelos e testes passaram a ser implementados tanto para tratar a questão dessas variáveis em nível quanto em seu segundo momento, ou seja sua volatilidade. Classicamente, para analisar a volatilidade univariada os modelos mais utilizados são VAR, GARCH e suas várias especificações. Os modelos GARCH multivariados, por sua vez, criaram oportunidade de analisar efeitos cruzados de volatilidade (variâncias e correlações condicionais).

570 Rev. Bras. Finanças (Online), Rio de Janeiro, V15, No. 4, Oct. 2013@ (1) 
Grande parte da evidência empírica disponível tem tratado os mercados de ações e de câmbio considerando apenas seus valores em nível. Yang and Doong (2004) observam que há uma escassez de evidência empírica que se concentra sobre as ligações entre o segundo momentos da distribuição das variáveis, ou seja, da variância entre os ativos. Uma série de estudos têm examinado no entanto como a volatilidade de um mercado de ações se espalha para outros mercados de ações, ou entre diferentes ativos como por exemplo a volatilidade do câmbio. Phylaktis and Ravazzolo (2005) mostram, a partir da aplicação da metodologia de cointegração e teste multivariado de causalidade de Granger, feitos a um grupo de países da Bacia do Pacífico, que os mercados de ações e de câmbio estão positivamente relacionadas.

Outra linha de estudo identificou efeitos de transbordamentos entre mercados financeiros internacionais como apresentado o estudo de citetHamao1990. Nesse estudo cotações diárias de abertura e fechamento dos principais índices de ações para os mercados de ações de Tóquio, Londres e Nova Iorque foram examinadas. A análise utiliza a família de modelos ARCH para explorar essas relações. Evidência de spillovers de volatilidade encontrada, tinha a direção dos preços de Nova Iorque para Tóquio, Londres a Tóquio e Nova York a Londres, contudo as volatilidades dos preços em outras direções foram encontradas para o período de pré-outubro de 1987.

O presente estudo contribui com a literatura sendo o primeiro artigo analisando a temática para o caso brasileiro, além ser pioneiro combinando dois diferentes tipos volatilidades (cambiais e de mercados financeiros internacionais) e seus transbordamentos para o mercado acionário brasileiro. O estudo ainda contribui utilizando, além dos modelos GARCH multivariados, testes de causalidade de Granger de segunda ordem para identificação da direção dos transbordamentos citados. $\mathrm{O}$ artigo é organizado apresentando na seção 2 um referêncial teórico com estudos relevantes sobre o tema, a seção 3 apresenta a descrição da metodologia utilizada: o modelo GARCH multivariado utilizado, um breve resumo do teste de causalidade de Granger de segunda ordem, o detalhamento da base de dados utilizada, a construção dos choques cambiais e de mercados financeiros internacionais e como serão organizados os modelos e testes implementados. Os resultados são apresentados na seção 4 com as con- 
clusões sendo apresentadas na seção 5.

\section{Referêncial Teórico}

\subsection{Spillover entre Volatilidade dos Preços de Ações e das Taxas de Câmbio}

Kanas (2000) foi um dos primeiros estudos que analisaram spillovers de volatilidade do retorno das ações à variação cambial nos EUA, Reino Unido, Japão, Alemanha, França e Canadá. Ele encontrou evidências de spillovers de retornos de ações à variação cambial para todos os países, exceto na Alemanha, o que sugere que a abordagem de ativos para a determinação da taxa de câmbio é válida quando formulada em termos do segundo momento da distribuição da taxa de câmbio para os países incluídos em sua análise. Spillovers Volatilidade de variações cambiais para os retornos das ações foram insignificantes para todos os países.

Yang and Doong (2004) exploraram a natureza do mecanismo de transmissão de média e volatilidade entre os mercados de ações e de divisas para os países do G-7. Eles expandiram a amostra Kanas (1998) ao G-7, incluindo a Itália. Os resultados apontaram para spillovers volatilidade significativa e um efeito assimétrico do mercado de ações para o mercado de câmbio para a França, Itália, Japão e os EUA, o que sugere a integração entre os mercados de ações e de câmbio nesses países. Sua evidência empírica mostrou que os movimentos de preços de ações terão um impacto sobre os movimentos futuros da taxa de câmbio, mas as mudanças nas taxas de câmbio têm menos efeito direto sobre futuros retornos de ações, que é similar aos achados de Kanas.

Em um estudo semelhante feito por Fedorova and Saleem (2009), entre quatro países do leste da Europa, incluíram em seu estudo, Polônia, Hungria e Rússia e mostraram evidências de efeitos colaterais unidirecionais de volatilidade de mercado de moeda para o mercado de ações na economia, e só a República Checa que mostraram os efeitos da volatilidade spillover bidirecionais entre os mercados.

Goldberg (1993) encontrou evidências para os EUA, de que mudanças na volatilidade da taxa de câmbio tem efeitos significativamente negativos a longo prazo sobre o investimento. Darby et al. (1999), usando uma estimativa com uma única equação em seu estudo, encontrou um efeito cambial negativo semelhante sobre o in-

572 Rev. Bras. Finanças (Online), Rio de Janeiro, V15, No. 4, Oct. 201 @ ( 
vestimento agregado com base nos dados de cinco países da OCDE. Carruth et al. (2000), adotando uma estrutura GARCH, encontrou um impacto altamente negativo significativo da incerteza da taxa de câmbio real sobre o investimento.

Amihud (1994) e Bartov and Bodnar (1994) constataram que as mudanças contemporâneas do dólar têm pouco poder para explicar retornos anormais. Eles também encontraram que uma mudança defasada do dólar está negativamente associada com retornos anormais. Os resultados da regressão mostraram que uma mudança defasada do dólar tem poder explicativo no que diz respeito a erros nas previsões de analistas de lucro trimestral.

Ajayi and Mougoue (1996) encontraram evidências de que variação cambial exerce uma influência significativa e dinâmica em retornos para oito países industrializados. Eles mostraram que um aumento no valor agregado doméstico das ações tem um efeito negativo de curto prazo no valor da moeda nacional, mas nos aumentos de longo prazo nos preços das ações têm um efeito positivo no valor da moeda doméstica. No entanto, a depreciação da moeda tem um efeito negativo de curto prazo no mercado de ações.

Zapatero (1995) mostra que, nos mercados financeiros totalmente integrados, há uma ligação explícita entre a volatilidade dos preços das ações e a volatilidade da taxa de câmbio. Jorion (1990) e Booth and Rotenberg (1990) não conseguiram encontrar uma ligação significativa entre a variação cambial e os retornos das ações de empresas dos EUA, e os EUA e empresas canadenses, respectivamente. Em um outro estudo semelhante Muradoglu et al. (2000) tentaram descobrir a relação entre retornos e algumas variáveis macroeconômicas e concluíram que existe uma relação causal da forma de taxas de câmbio para o retorno das ações na Nigéria, México, Coreia, Grécia , Colômbia e Brasil.

Chen et al. (2004) apontaram que em um grande mercado com empresas bem diversificadas, fatores domésticos podem ser mais importantes do que os fatores internacionais. Em contrapartida, o mercado da Nova Zelândia é muito pequeno em relação ao mercado norteamericano e as empresas na Nova Zelândia são muito menos diversificadas. Usando um modelo de dois fatores, Chen et al. (2004) acharam que os retornos de empresas da Nova Zelândia (o primeiro momento) são significativamente explicados pela variação cambial. No entanto,

(9) (1) Rev. Bras. Finanças (Online), Rio de Janeiro, V15, No. 4, Oct. 2017573 
não analisaram a volatilidade (segundo momento) spillover entre os retornos do mercado de ações e mudanças nas taxas de câmbio na economia da Nova Zelândia.

Black (1976) e Christie (1982) perceberam que uma queda do preço das ações é seguido por um aumento na volatilidade das ações subsequente. Esta observação é denominada como o efeito de alavanca, que é testado no modelo GJR-GARCH desenvolvido por Glosten et al. (1993).

Um estudo de Alaganar and Bhar (2007) indica que os efeitos de primeira e de segunda ordem da taxa de câmbio têm um impacto significativo em carteiras diversificadas no mercado acionário norte-americano. Empregaram os modelos GJR-GARCH e GARCHM para testar o impacto da volatilidade da taxa de câmbio sobre os retornos de carteiras de índices de país diversificados. Eles apontaram que a variância da taxa de câmbio é importante para diversificações no mercado de ações.

Morales (2008) estudou os spillovers Volatilidade entre retornos e de câmbio: evidências de quatro países do Leste Europeu. Para os efeitos de transbordamento assimétricos eles descobriram que este efeito é relevante para todos os países, a partir dos retornos das ações para as taxas de câmbio, tendo todos os coeficientes com sinais positivos, que é interpretada da seguinte forma: uma boa notícia tem um impacto maior na volatilidade do que inesperada má notícia.

Diamandis and Drakos (2011) examinaram a dinâmica de longo prazo e de curto prazo entre os mercados de ações e câmbio de quatro países latino-americanos (Argentina, Brasil, Chile e México), bem como suas interações com os mercados de ações dos EUA. Esses autores encontraram que os dois mercados nessas economias estão positivamente relacionados e o mercado de ações dos Estados Unidos representa um canal de transmissão para esses efeitos.

Chkili and Nguyen (2014) usaram uma abordagem de modelo de mudança de regime para investigar as relações dinâmicas entre as taxas de câmbio e os retornos do mercado de ações para os países BRICS (Brasil, Rússia, Índia, China e África do Sul). A análise univariada indica que os retornos das ações dos países do BRICS evoluem de acordo com dois regimes diferentes: um regime de baixa volatilidade e um regime de alta volatilidade. Por outro lado, os modelos VAR com mudança de regime markoviano sugerem que os mercados

574 Rev. Bras. Finanças (Online), Rio de Janeiro, V15, No. 4, Oct. 201 @ (๑) 
de ações têm mais influência nas taxas de câmbio durante os dois períodos calmos e turbulentos. Estas percepções empíricas têm implicações importantes para os investimentos em carteira e cobertura de risco cambial.

\subsection{Volatilidade entre Índices do Mercado Financeiro}

A interdependência de curto prazo dos preços e da volatilidade dos preços através de três principais mercados de ações internacionais foram estudadas por Hamao et al. (1990). Cotações diárias de abertura e fechamento dos principais índices de ações para os mercados de ações de Tóquio, Londres e Nova Iorque foram examinadas. A análise utiliza a família auto-regressivo com heterocedasticidade condicional $(\mathrm{ARCH})$ para explorar essas relações de preços. Evidência de spillovers de volatilidade dos preços de Nova Iorque para Tóquio, Londres a Tóquio e Nova York a Londres foram observadas, mas sem efeitos colaterais as volatilidades dos preços em outras direções foram encontradas para o período de pré-outubro de 1987.

O resultado da pesquisa de Theodossiou and Lee (1993) resultou em artigo que trouxe esclarecimentos adicionais sobre a natureza e o grau de interdependência dos mercados de ações dos Estados Unidos, Japão, Reino Unido, Canadá e Alemanha, e relatou o grau em que a volatilidade nesses mercados influenciam retornos esperados. A análise utilizou o modelo GARCH-M multivariado. Apesar de serem considerados fracos, spillovers estatisticamente significativos irradiaram de mercados de ações dos EUA para o Reino Unido, Canadá e Alemanha, e depois dos mercados de ações do Japão para a Alemanha. Nenhuma relação foi encontrada entre a volatilidade do mercado condicional e retornos esperados. Forte volatilidade condicional, variável no tempo, foi encontrada na série de retorno de todos os mercados. Os spillovers de volatilidade nos próprios mercados do Reino Unido e do Canadá são insignificantes, suportando a ideia de que a volatilidade condicional dos retornos nesses mercados é "importado"do exterior, especialmente dos Estados Unidos onde spillovers de volatilidade irradiaram significativamente do mercado de ações dos EUA para os quatro mercados de ações, do mercado de ações do Reino Unido para o mercado de ações canadense, e do mercado de ações alemão para o mercado de ações japonês. Os resultados encontrados foram robustos e não ocorreram alterações na estrutura

(@) (๑) Rev. Bras. Finanças (Online), Rio de Janeiro, V15, No. 4, Oct. 2017575 
de correlação dos retornos ao longo do tempo.

O artigo de Susmel and Engle (1994) examinou os spillovers de volatilidade entre Nova Iorque e os mercados de ações de Londres. Usando um modelo ARCH verificaram que a evidência de spillovers de volatilidade entre estes mercados é mínima e têm uma duração de uma hora, ou menos. Os efeitos mais significativos envolvem o movimento dos preços das ações em torno da abertura de Nova Iorque, mas esses resultados não são fortes.

O artigo de Wei et al. (1995) testou a sabedoria convencional de que a volatilidade de preços e mudanças a curto prazo transbordam dos países desenvolvidos para os mercados emergentes, mas não vice e versa. Os autores também investigaram como o grau de abertura do mercado afeta os retornos e transbordamentos de volatilidade. Três mercados desenvolvidos, Nova Iorque, Tóquio e Londres, e dois mercados emergentes, Taiwan e Hong Kong, foram examinados. Dois achados mais interessantes foram: em primeiro lugar, o mercado de Tóquio tem menos influência do que o mercado de Nova Iorque sobre os mercados de Taiwan e Hong Kong; e segundo, o mercado de Taiwan é mais sensível do que o mercado de Hong Kong para o comportamento dos preços e volatilidade dos mercados avançados, apesar de Taiwan não ser tão aberto como Hong Kong e o dólar de Taiwan não estar ligado ao dólar americano, enquanto o dólar de Hong Kong está.

Veiga and McAleer (2003) testaram a existência de spillovers de preços e de volatilidade entre os três principais índices do mercado de ações, ou seja, S\&P 500, FTSE 100 e Nikkei 225. O VARMAAGARCH modelo de Hoti et al. (2002) foi usado para modelar as volatilidades condicionais multivariadas e para testar a existência de spillovers de volatilidade. Este modelo geral não foi aplicado anteriormente para testar transbordamentos de volatilidade.O modelo VARMA-AGARCH e a estimativa sequencial permitem obter uma inferência estatística válida porque as propriedades estruturais e estatísticas do modelo foram estabelecidas em Hoti et al. (2002).

Em Veiga and McAleer (2003) evidência significativa de spillovers de retornos foram encontrados em todos os pares de índices de ações, bem como spillovers de volatilidade a partir FTSE 100 a ambos S\&P 500 e Nikkei 225, e de S\&P 500 e FTSE 100. Estes resultados diferem dos da literatura, em que a volatilidade originária da S\&P 500 tem 
sido geralmente encontrados para ter transbordamento a todos os outros índices, e foi encontrado resultado da volatilidade de Nikkei 225 tendo efeitos (spillovers) para FTSE 100.

Miyakoshi (2003) em seu artigo examinou a magnitude do retorno e volatilidade spillovers do Japão e dos EUA a sete mercados de ações asiáticos. Foi construído um modelo spillover de volatilidade para lidar com o choque dos EUA como uma variável exógena em um EGARCH bivariado para o Japão e para os mercados asiáticos. Em primeiro lugar, apenas a influência dos EUA foi considerada importante para os retornos do mercado da Ásia; não foi identificada influência do Japão. Em segundo lugar, a volatilidade do mercado asiático foi mais influenciada pelo mercado japonês que pelos EUA. Em terceiro lugar, foi observada uma influência negativa da volatilidade do mercado asiático para o mercado japonês.

Baele (2005)investigaram em que medida a globalização afetou a crescente interdependência do mercado de ações. Concentraram-se na Europa Ocidental, já que esta região passou por um período único de integração econômica, financeira e monetária. Mais especificamente, os autores quantificam a magnitude e os efeitos de contágio de volatilidade do mercado agregado europeu e do mercado dos EUA para 13 mercados de ações europeus locais. Para ter em conta a integração variável no tempo, foi utilizado um modelo de mudança de regime para permitir que as sensibilidades à mudança de choque ao longo do tempo fossem identificadas. Tanto a intensidade do choque spillover do agregado europeu quanto dos EUA aumentaram substancialmente ao longo dos anos 1980 e 1990, embora o aumento tenha sido mais pronunciado para spillovers do agregado europeu.

Em uma análise de 19 mercados globais de capital a partir do início de 1990 até 2009, Diebold and Yilmaz (2009) encontraram evidência notável de comportamento divergente na dinâmica de spillovers de retorno versus spillovers de volatilidade: spillovers de retorno exibem uma tendência ligeiramente crescente, enquanto spillovers de volatilidade não exibem tendência, mas saltos claros. Diebold and Yilmaz (2009) forneceram uma medida simples e intuitiva de interdependência dos retornos de ativos e/ou de volatilidades. Em particular, formularam e analisaram as medidas precisas e separadas de spillovers de retorno e de volatilidade. A estrutura utilizada facilitou o estudo de ambos, não de crise e episódios de crise, incluindo 
tendências e quebras em spillovers; ambos acabam por ser empiricamente importantes.

Foram estudadas por Bae and Karolyi (1994) a dinâmica conjunta de volatilidade de retorno durante a noite e durante o dia para o Nikkei Stock Average em Tóquio e a Standard and Poor Stock Index 500 em Nova Iorque no período 1988-1992. Os autores estenderam a estrutura GARCH de Engle (1982) e Bollerslev (1986) para permitir efeitos negativos assimétricos ("más notícias") e positivos ("boas notícias"). A evidência mostrou que a magnitude e persistência dos choques originários de Nova Iorque ou de Tóquio que transmitem a outro mercado sejam compreendidos significantemente, se esse efeito assimétrico for ignorado.

O artigo de Kanas (1998) examinou a questão dos spillovers de volatilidade nos três maiores mercados de ações europeus, nomeadamente Londres, Frankfurt e Paris. O modelo auto-regressivo com heterocedasticidade condicional (ARCH) foi usado para capturar potenciais efeitos assimétricos de inovações sobre a volatilidade. Durante o período de 01/01/84 a 07/12/93, spillovers recíprocas são encontrados para existir entre Londres e Paris, e entre Paris e Frankfurt, e spillovers unidirecionais de Londres para Frankfurt. Em quase todos os casos, as repercussões são assimétricas no sentido de que más notícias em um mercado tem um efeito maior sobre a volatilidade de um outro mercado do que uma boa notícia.

\section{Metodologia}

\subsection{Modelo GARCH Multivariado BEKK}

Os modelos de séries de tempo univariados foram pioneiros nos estudos da volatilidade do retorno de ativos financeiros, em especial quando essa volatilidade tem um comportamento variando ao longo do tempo. Esses estudo evoluíram para os casos multivariados. Nesta subseção vamos apresentar alguns modelos apropriados para séries financeiras que apresentam a variância condicional evoluindo no tempo. Existe uma variedade grande de modelos não-lineares disponíveis na literatura, aqui iremos nos concentrar em algumas extensões da classe de modelos ARCH (auto-regressivos com heteroscedasticidade condicional), introduzida por Engle (1982).

Uma generalização dos modelos ARCH foi sugerida por Bollerslev (1986), o chamado modelo GARCH ("generalized ARCH"). Um

578 Rev. Bras. Finanças (Online), Rio de Janeiro, V15, No. 4, Oct. 2017@ (용 
modelo GARCH pode ser usado para descrever a volatilidade com menos parâmetros do que um modelo ARCH.

Contudo em muitas situações de pesquisa existe a necessidade de considerarmos mais de um ativo, e consequentemente suas correlações. Dessa forma, assim como a volatilidade variando no tempo ganhou repercussão em pesquisas, a correlação variando começou a ganhar destaques com os modelos GARCH multivariados (MGARCH).

O modelo GARCH multivariado tem a seguinte forma geral:

$$
\begin{array}{r}
\operatorname{vech}\left(\Sigma_{t \mid t-1}\right)=A_{0}+\sum_{j=1}^{q} A_{j} \operatorname{vech}\left(u_{t-j} u_{t-j}^{\prime}\right)+\sum_{j=1}^{m} B_{j} \operatorname{vech}\left(\Sigma_{t-j \mid t-j-1}\right) \\
u_{t}=\Sigma_{t}^{1 / 2} \mathbf{z}_{t}, \mathbf{z}_{t} \sim i i d\left(\mathbf{0}, \mathbf{I}_{K}\right) \\
\Sigma_{t}=\Sigma_{t}^{1 / 2}\left(\Sigma_{t}^{1 / 2}\right) \quad(\text { Cholesky })
\end{array}
$$

O espaço de parâmetros de um modelo MGARCH tem uma dimensão grande e, em geral, precisa ser restringido para garantir a unicidade da representação e obter propriedades adequadas de covariâncias condicionais. Para reduzir o espaço de parâmetros, Bollerslev et al. (1988) discutiram modelos MGARCH diagonais, onde o $A_{j}{ }^{\prime} s$ e $B_{i}{ }^{\prime} s$ são matrizes diagonais. Em alternativa, um modelo BEKK GARCH é usualmente representado da seguinte forma:

$$
\Sigma_{t \mid t-1}=A_{0}^{* \prime} A_{0}^{*}+\sum_{n=1}^{N} \sum_{j=1}^{q} A_{j n}^{*^{\prime}} u_{t-j} u_{t-j}^{\prime} A_{j n}^{*}+\sum_{n=1}^{N} \sum_{j=1}^{m} B_{j n}^{*^{\prime}} \Sigma_{t-j \mid t-j-1} B_{j n}^{*}
$$

Onde $A_{0}^{*}$ é uma matriz triangular $K \times K$ e os coeficientes matriciais $A_{j n}^{*^{\prime}}$ e $B_{j n}^{*^{\prime}}$ são também $K \times K$.

Embora o modelo de BEKK de baixa ordem seja uma representação relativamente parcimoniosa da estrutura de covariâncias condicionais, o número de parâmetros ainda cresce rapidamente com a dimensão do sistema subjacente. Portanto, na prática, é apenas factível se sistemas com apenas algumas variáveis estão sendo considerados e outras medidas de simplificação foram propostas para aliviar a modelagem de processos de dimensões mais elevadas. Alguns deles podem ser vistos como modelos especiais BEKK.

Nos mercados financeiros, tem sido observado com frequência que os choques negativos e positivos, ou notícias, têm efeitos muito diferentes Black (1976). Esse efeito de alavanca pode ser introduzido de forma diferente em modelos MGARCH. Por exemplo, Hafner and

(9) (1) Rev. Bras. Finanças (Online), Rio de Janeiro, V15, No. 4, Oct. 2017579 
Herwartz (1998) e Herwartz and Lütkepohl (2000) generalizaram uma proposta univariada de ? e substituíram $A_{11}^{* \prime} u_{t-1} u_{t-1}^{\prime} A_{11}^{*}$ por

$$
A_{11}^{*^{\prime}} u_{t-1} u_{t-1}^{\prime} A_{11}^{*}+A_{-}^{*^{\prime}} u_{t-1} u_{t-1}^{\prime} A_{-}^{*} I\left(\sum_{k=1}^{K} u_{k t}<0\right)
$$

em um modelo BEKK com $\mathrm{N}=1$.

Aqui I(.) denota uma função indicadora que assume o valor 1 se o argumento é válido e 0 se não e $A_{-}^{*}$ é um coeficiente adicional da matriz $(K \times K)$.

\subsection{Teste de Causalidade de Granger de Segunda Ordem}

A definição de causalidade de Granger se baseia em previsões. Sob condições adequadas, as previsões ótimas são obtidas como esperanças condicionais. Portanto, a causalidade de Granger pode ser definida em termos ótimos como esperanças condicionais. Em outras palavras, podemos definir uma variável de séries de tempo $X_{t}$ para ser causal de $Z_{t}$, se

$$
E\left(z_{t+1} \mid z_{t}, z_{t-1, \ldots}\right) \neq E\left(z_{t+1} \mid z_{t}, z_{t-1}, \ldots, x_{t}, x_{t-1}, \ldots\right) .
$$

Esta definição sugere uma extensão direta de ordem superior de momentos condicionais. Nós definimos $x_{t}$ ser causal para $z_{t}$ no résimo momento se

$$
E\left(z_{t+1}^{r} \mid z_{t}, z_{t-1, \ldots}\right) \neq E\left(z_{t+1}^{r} \mid z_{t}, z_{t-1}, \ldots, x_{t}, x_{t-1}, \ldots\right) .
$$

Assim, a primeira desigualdade define a causalidade em média e considerando os segundos momentos centrais a segunda desigualdade dá uma definição de causalidade na variância que é análoga à definição prévia de causalidade de Granger em média. Em outras palavras, se $X_{t}$ é causal na variância para $Z_{t}$, a volatilidade condicional de $Z_{t}$ pode ser prevista de forma mais precisa, tendo em conta a informação presente e passada em $X_{t}$ sem levar em conta esta informação.

Um vetor de variáveis não causa a outro vetor de variáveis, conforme a causalidade de Granger de segunda ordem, se as informações

580 Rev. Bras. Finanças (Online), Rio de Janeiro, V15, No. 4, Oct. 201 
do passado sobre a variabilidade do primeiro não pode melhorar a previsão de variâncias condicionais dos últimos. A definição da não causalidade de segunda ordem assume que as relações causais de Granger podem existir no processo de média condicional, no entanto, elas devem ser modeladas a partir de filtros. Caso contrário, essas relações podem ter impacto sobre os parâmetros responsáveis pelas relações causais em variâncias condicionais.

\subsection{Teste $\mathbf{H H}$}

Com base nos quadrados dos resíduos $\hat{\xi}_{i, t}^{2}=u_{i, t}^{2} / \hat{\sigma}_{i, t}^{2}$ onde $\hat{\sigma}_{i, t}^{2}$ é a variância condicional estimada de $u_{i, t}$ usando GARCH univariado, Cheung and Ng (1996) introduziram uma estatística manual para testar a hipótese nula de não causalidade na variância. Na prática, a escolha de $m$ deve permitir cobrir o maior potencial de defasagem da casualidade na variância. Cheung and $\mathrm{Ng}$ (1996) provaram que sob estimativa consistente de parâmetros GARCH univariadas $P_{m}$ segue assintoticamente a distribuição $\chi_{m}^{2}$ sob a hipótese nula. Estatísticas análogas podem ser definidos para testar a hipótese de causalidade bidireccional. Uma versão multivariada foi proposta por Hafner and Herwartz (2008), conforme apresentaremos a seguir.

A não causalidade em variância está associada a um certo conjunto de restrições que zeram alguns valores das matrizes $A_{j}$ e $B_{j}$ em

$$
\operatorname{vech}\left(\Sigma_{t \mid t-1}\right)=A_{0}+\sum_{j=1}^{q} A_{j} \operatorname{vech}\left(u_{t-j} u_{t-j}^{\prime}\right)+\sum_{j=1}^{m} B_{j} \operatorname{vech}\left(\Sigma_{t-j \mid t-j-1}\right)
$$

Para encontrar essas restrições, primeiramente definimos o índice

$$
k_{i j}^{K}=i+(j-1)\left(K-\frac{j}{2}\right)
$$

para $i j \in \tau \cup v$ e $i \geq j$ que é a posição do $(i, j)$-th elemento da matriz M simétrica $(K \times K)$ no vetor vech $(M)$. Lembrando que $\operatorname{vech}(M)$ contém $K^{*}=\frac{K(K+1)}{2}$ elementos. Além disso, definimos os conjuntos de índices

$$
\tau^{*}=\left\{k_{i j}^{K} \mid i, j \in \tau\right\}
$$

(9) (๑) Rev. Bras. Finanças (Online), Rio de Janeiro, V15, No. 4, Oct. 2017581 


$$
v^{*}=\left\{1, \ldots, K^{*}\right\} \mid \tau^{*} .
$$

A partir dessas notações, podemos agora definir as condições para a não causalidade na variância. Consideremos as duas condições a seguir

$$
\begin{gathered}
{\left[\Phi_{n}\right]_{i j}=0 .} \\
\forall n \geq 1, \forall i \in \tau^{*}, \forall j \in v^{*} .
\end{gathered}
$$

e

$$
\begin{gathered}
{\left[A_{a}\right]_{i j}=0 .} \\
a=1, \ldots, q \\
{\left[B_{b}\right]_{i j}=0 .} \\
b=1, \ldots, p, \forall i \in \tau^{*}, \forall j \in v^{*} .
\end{gathered}
$$

Supomos agora que $\tilde{Q}$ é uma matriz de dimensão $k(K-k) \times(K)^{2}$, de posto $k(K-k)$. Os $(r, \varpi)$ elementos de $\tilde{Q}$ são definidos por

$$
\tilde{Q}_{r, \varpi}=\left\{\begin{array}{l}
1, \varpi=s_{m n} \\
0, \varpi \neq s_{m n}
\end{array}\right.
$$

Onde $r=m+(n-1), s_{m n}=i_{m}+\left(j_{n}-1\right) K, i_{m} \in \tau, j_{n} \in v$, e $m=1, \ldots, k, n=1, \ldots, K-k$.

A hipótese nula de ausência de causalidade no modelo BEKK agora pode ser escrita como

$$
H_{0}: Q v=0
$$

582 Rev. Bras. Finanças (Online), Rio de Janeiro, V15, No. 4, Oct. 201 
$\operatorname{Com} v=\left[\operatorname{vech}\left(A_{0}^{*}\right), \operatorname{vech}\left(A_{j n}^{*}\right)^{\prime}, \operatorname{vech}\left(B_{j n}^{*}\right)\right]^{\prime} \mathrm{e}$ $Q=\left[0_{k(K-k) \times(K)}, \tilde{Q}, \tilde{Q}\right]$.

Suponha agora que temos $\mathrm{T}$ observações $u_{1}, \ldots, u_{T}$. Nós assumimos a seguir que o verdadeiro processo é conhecido como pertencendo à classe BEKK, conforme Comte and Lieberman (2003). Denotamos um estimador consistente do verdadeiro vetor de parâmetros $\vartheta_{0}$ por $\hat{\vartheta}$ e assumimos que a sua distribuição assintótica é dada por

$$
\sqrt{T}\left(\hat{\vartheta}-\vartheta_{0}\right) \stackrel{\text { ass }}{\longrightarrow} N\left(0, \Omega_{\vartheta}\right)
$$

Com alguma matriz definida e simétrica positiva $\Omega_{\vartheta}$. Consideremos também que uma estimador consistente de $\Omega_{\vartheta}$ é dado por $\hat{\Omega_{\vartheta}}$. Então $\sqrt{T}\left(\hat{\vartheta}-\vartheta_{0}\right) \stackrel{\ell}{\longrightarrow} N\left(0, \Omega_{\vartheta}\right)$ mantém em condições de regularidade listados por Comte and Lieberman (2003), e $\Omega_{\vartheta}$ é dado por

$$
\Omega_{\vartheta}=S^{-1} D S^{-1}
$$

Onde

$$
D=E\left[\left.\frac{\partial l_{t}(\vartheta)}{\partial \vartheta} \frac{\partial l_{t}(\vartheta)}{\partial \vartheta^{\prime}}\right|_{\vartheta_{0}}\right], S=-E\left[\left.\frac{\partial^{2} l_{t}(\vartheta)}{\partial \vartheta \partial \vartheta^{\prime}}\right|_{\vartheta_{0}}\right],
$$

Com

$$
l_{t}(\vartheta)=-\frac{K}{2} \ln (2 \pi)-\frac{1}{2} \ln \left|\Sigma_{t \mid t-1}(\vartheta)\right|-\frac{1}{2} v_{t}^{\prime} \Sigma_{t \mid t-1}^{-1}(\vartheta) v_{t}
$$

Hafner and Herwartz (2008) forneceram expressões para $D$ e $S$ e de suas estimativas. Para os testes de significância Hafner and Herwartz (2008) mostram que a utilização de técnicas analíticas expressões para $\Omega_{\vartheta}$ é de longe superior ao uso de derivados numéricos em termos de tamanho empírico e poder de estimativas.

Propomos a seguinte estatística Wald padrão para testar as hipóteses $H_{0}: Q v=0$,

$$
W_{T}=T(Q \hat{\vartheta})^{\prime}\left(Q \hat{H}_{\vartheta} Q^{\prime}\right)^{-1}(Q \hat{\vartheta})
$$


Usando $\sqrt{T}\left(\hat{\vartheta}-\vartheta_{0}\right) \stackrel{\text { ass }}{\longrightarrow} N\left(0, \Omega_{\vartheta}\right)$ e a proposição de Lütkepohl (1993), a distribuição assintótica da estatística Wald é dada por

$$
W_{T} \stackrel{a s s}{\longrightarrow} \chi_{k(K-k)}^{2}
$$

Uma estatística análoga pode ser definida para o modelo Diagonal VEC com base na hipótese nula $H_{0}: Q v=0$, desde que as condições de normalidade assintótica dos estimadores forem atendidas. Notese que os graus de liberdade da estatística de Wald para o modelo Diagonal VEC seria $k^{*}\left(K^{*}-k^{*}\right)$.

\subsection{Descrição dos Modelos, Análise de Componentes Prin- cipais e Testes Utilizados}

Uma relevante questão é a definição de choques de volatilidades cambiais e de mercados financeiros internacionais. Muito embora muitas definições possam ser adaptadas para capturar tais volatilidades, o presente estudo contou com a série temporal ${ }^{1}$ de 48 taxas cambias e 17 índices de bolsas de valores além do Ibovespa, no período amostral de 02/01/2007 a 31/03/2016, diariamente ${ }^{2}$. A proposta para tais choques cambiais e de mercados financeiros internacionais, visa construir um retorno representativo a partir dos conjuntos de informação:

- IND1 - volatilidades de 48 câmbios: foram utilizados 48 câmbios com o intuito de capturar um efeito generalizado de choques cambiais.

- IND2 - volatilidades de 47 câmbios (excluindo-se o Dólar/Real): nesse conjunto de dados, com a exclusão do dólar, buscamos estimar um efeito contrafactual de todas as demais taxas de câmbio.

- IND3 - volatilidades de 17 bolsas de valores: foram utilizados 17 índices de bolsas de valores com o intuito de capturar um efeito generalizado de choques de mercados financeiros internacionais.

\footnotetext{
${ }^{1} \mathrm{~A}$ tabela com as 48 taxas cambiais utilizadas e 17 Bolsas de Valores, estão descritas no Apendice.

${ }^{2}$ Todos os dados utilizados estão disponíveis e foram obtidos na plataforma Bloomberg, muito embora o número de dados faltantes não represente mais de 0,5\% dos dados, foi utilizado o algoritmo EM para estimação e imputação desses dados faltantes conforme detalhado em DiCesare (2006).
}

584 Rev. Bras. Finanças (Online), Rio de Janeiro, V15, No. 4, Oct. 201)@ (1) 
- Dólar - volatilidade do Dólar: objetivando capturar os efeitos únicos do dólar dado sua importância como principal moeda que compõe as reservas internacionais.

Os retornos foram calculados para cada série de dados de cada um dos conjuntos de informação. A partir dos retornos calculados foi utilizada a metodologia de análise de componentes principais (ACP), para cada conjunto, a fim de obter um índice de retorno que fosse representativo da variabilidade. Claramente, a exceção desse tratamento com ACP é o conjunto de dados do dólar, o qual foi calculado apenas o retornos do Dólar/Real. dos retornos dos dados em questão. Os índices representativos foram denominados IND1, IND2 e IND3.

Os 3 modelos estimados seguiram a lógica de combinar uma variá vel resposta Ibovespa com um índice que espelhe choque cambial (IND1, IND2 ou Retorno do Dólar) e o que representa choque de mercado financeiro internacional (IND3).

Para cada modelo foram estimadas as correlações condicionais com o modelo MGARCH BEKK, essa escolha se deve ao fato de que o teste HH está implementado para a utilização dos resultados desses modelos. Utilizamos testes de auto-correlação para os resíduos obtidos desses modelos para verificar sua adequação. Esses testes foram aplicados para os resíduos padronizados e para o quadrado dos resíduos padronizados. Foram feitos os testes de causalidade de Granger de segunda ordem (Teste HH) para identificar a direção da causalidade, no sentido de Granger, das correlações condicionais estimadas.

\section{Resultados}

Inicialmente observamos se os conjuntos de dados poderiam ser tratados por meio de ACP. Para tanto, em termos exploratórios, observamos como adequado o uso de ACP por meio da estatística KMO com valores acima de 0,9. Em termos inferenciais o teste de Bartlet indicou adequada utilização do ACP, com a rejeição da hipótese nula.

Conforme observamos a primeira componente, em todos os casos, tem alta representatividade em termos da variância total. Na sequência apresentamos os resultados das estatísticas exploratórias.

Os resultados da tabela 2 indicam a presença de fatos estilizados de retornos elencados por Caldeira et al. (2010), como média próxima

(@) (๑) Rev. Bras. Finanças (Online), Rio de Janeiro, V15, No. 4, Oct. 2017585 
Tabela 1

Resultados do Teste de Bartlet e Estatística KMO

\begin{tabular}{ccccc}
\hline Índices & Descrição & $\begin{array}{c}\text { BARTS } \\
\text { P-Valor }\end{array}$ & KMO & $\begin{array}{c}\text { Var. Explicada } \\
1^{\circ} \text { Comp. Principal }\end{array}$ \\
\hline IND1 & CAMBIOS - RET. & 0,00 & 0,97 & 0,884 \\
IND2 & CAMBIOS - RET. SEM DOLAR & 0,00 & 0,97 & 0,885 \\
IND3 & BOLSAS - RET. & 0,00 & 0,90 & 0,886 \\
\hline & $H_{0}$ : Não é adequado o uso da Análise de Componentes Principais ou Análise Fatorial.
\end{tabular}

Valores de KMO entre $(0,5$ e 1,0) indicam que a análise fatorial ou análise de Componentes Principais é apropriada.

Tabela 2

Análise Exploratória - Retornos

\begin{tabular}{|c|c|c|c|c|c|}
\hline TESTES/ESTATÍSTICAS & IBOVESPA & IND1 & IND2 & IND3 & Dólar \\
\hline $\mathrm{n}$ & 2284 & 2284 & 2284 & 2284 & 2284 \\
\hline Média & 0,02 & $-0,01$ & 0,02 & 0,02 & 0,02 \\
\hline Mediana & 0,02 & $-0,01$ & 0,01 & 0,06 & 0,008 \\
\hline Máximo & 14,65 & 3,30 & 8,32 & 8,70 & 6,60 \\
\hline Minimo & $-11,39$ & $-9,71$ & $-4,47$ & $-13,75$ & $-7,30$ \\
\hline Variância & 3,47 & 0,34 & 0,47 & 2,18 & 1,26 \\
\hline Desvio Padrão & 1,86 & 0,58 & 0,69 & 1,47 & 1,12 \\
\hline Assimetria & 0,20 & $-2,46$ & 1,56 & $-0,76$ & 0,30 \\
\hline Curtose & 9,34 & 42,99 & 24,37 & 11,59 & 7,87 \\
\hline Coef. Variação & 86,17 & 40,51 & 24,90 & 60,56 & 38,52 \\
\hline Jarque $\left.\operatorname{Bera}_{(P-V a l o r}\right)$ & 0,00 & 0,00 & 0,00 & 0,00 & 0,00 \\
\hline $\operatorname{ADF}_{(P-\text { valor })}$ & 0,00 & 0,00 & 0,00 & 0,00 & 0,00 \\
\hline
\end{tabular}

de zero, presença de curtose e assimetria, ausência de normalidade e estacionaridade das séries. Esses resultados representam um primeiro indicativo de os retornos representativos obtidos via ACP de fato representam uma medida resumo de retornos de cambios e de mercados financeiros internacionais, todavia essa comprovação ocorre pela utilização do teste $\mathrm{LM}^{3}$ para retornos e quadrado dos retornos, ou seja, a confirmação de ausência de autocorrelação para retornos, mas presença de estrutura de autocorrelação no quadrado dos retornos, conforme apresentamos nos gráficos a seguir.

A seleção das ordens em modelos GARCH é mais desenvolvida para o caso univariado, não havendo extensa literatura para o caso multivariado conforme Lütkepohl (2005). Contudo, para este estudo utilizamos a abordagem de identificação do caso univariado como indicação para o caso multivariado haja vista que para o MGARCH es-

\footnotetext{
${ }^{3}$ Teste do Multiplicador de Lagrange para autocorrelação com a $H_{0}$ : Ausência de autocorrelação na k-ésima defasagem.
}

586 Rev. Bras. Finanças (Online), Rio de Janeiro, V15, No. 4, Oct. 201 @@ @ 


\section{Figura 1}

Teste LM para autocorrelação Para Retornos e Volatilidades

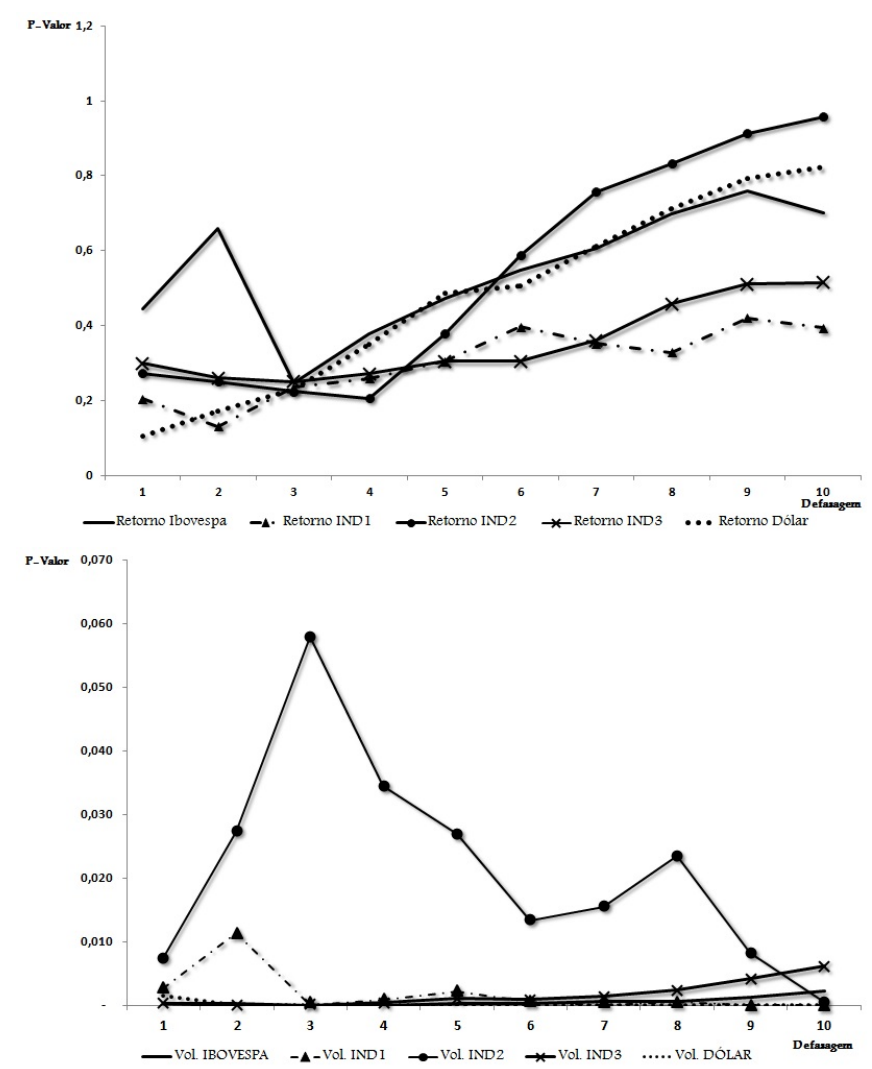

trutural (BEKK) a saturação de parâmetros pode rapidamente comprometer sua estimação. Dessa forma, utilizaremos a sequencia sugerida por Franses and Van Dijk (2000). Obtemos dessa forma, para o caso univariado os resultados que se seguem:

Com base nos resultados na tabela acima obtivemos, para todas as séries, que a ordem univariada sugerida foi de no máximo 2 , tanto para o componente de autocorrelação quanto para o componente de média móvel. Assim, tivemos a indicação de que as ordens dos modelos a serem estimados, para o caso multivariado, também fossem de ordem baixa. Contudo, a validação das ordens sugeridas se dará pela eliminação da autocorrelação dos resíduos padronizados e do quadrado dos resíduos padronizados.

(9) (-) Rev. Bras. Finanças (Online), Rio de Janeiro, V15, No. 4, Oct. 2017587 
Tabela 3

Identificação das possíveis ordens dos modelos

\begin{tabular}{ccc}
\hline Variáveis & Análise ACF/PACF & Ordem Sugerida \\
\hline Ibovespa & PACF2 & GARCH(1,1) ou GARCH $(1,2)$ \\
IND1 & PACF2 & GARCH $(1,1)$ ou GARCH $(1,2)$ \\
IND2 & ACF1 e PACF1 e Decaimento Exponencial & GARCH $(2,1)$ ou GARCH $(1,2)$ \\
IND3 & ACF2 e PACF2 & GARCH $(1,2)$ \\
Dólar & PACF2 & GARCH $(1,1)$ ou GARCH $(1,2)$ ou GARCH $(2,1)$ \\
\hline
\end{tabular}

\subsection{Modelos MGARCH-BEKK Estimados}

Nessa seção apresentamos os resultados dos modelos estimados que adequadamente reproduziram o processo gerador das séries. Conforme a indicação da tabela 3, os modelos GARCH multivariados apresentaram baixa ordem. 


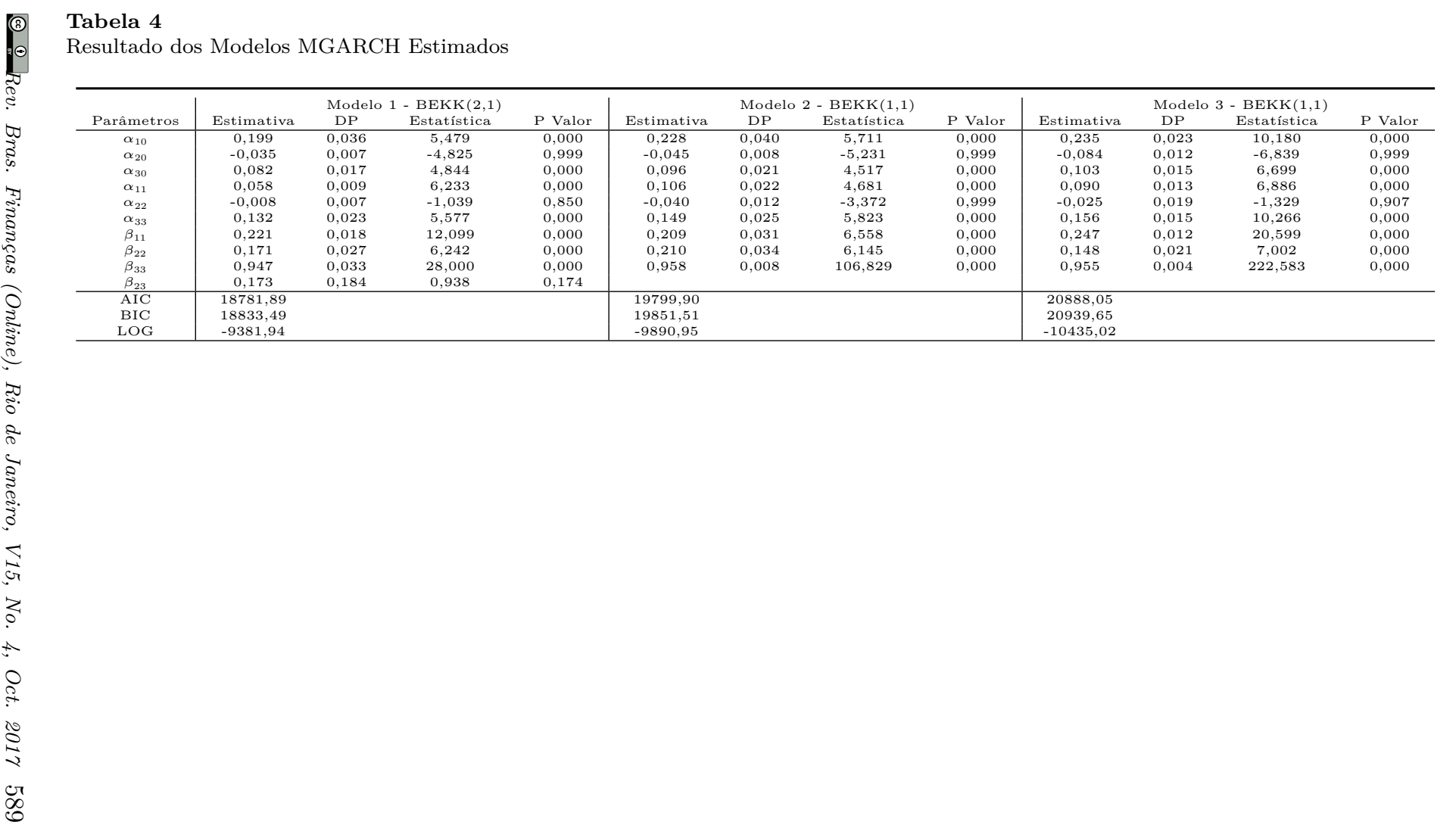


Os modelos apresentatam poucos parâmetros sem significância estatística, e nas ordem estimadas eliminaram a estrutura de autocorrelação dos resíduos e do quadrado dos resíduos, com excessão para os resíduos do modelo 2 (que considera todos os retornos de todas as taxas cambais exceto o dólar) associados a proxy de choque de mercados financeiros internacionais, conforme os gráficos a seguir.

\section{Figura 2}

Teste LM para autocorrelação Para Resíduos e Quadrado dos Resíduos - Modelo 1
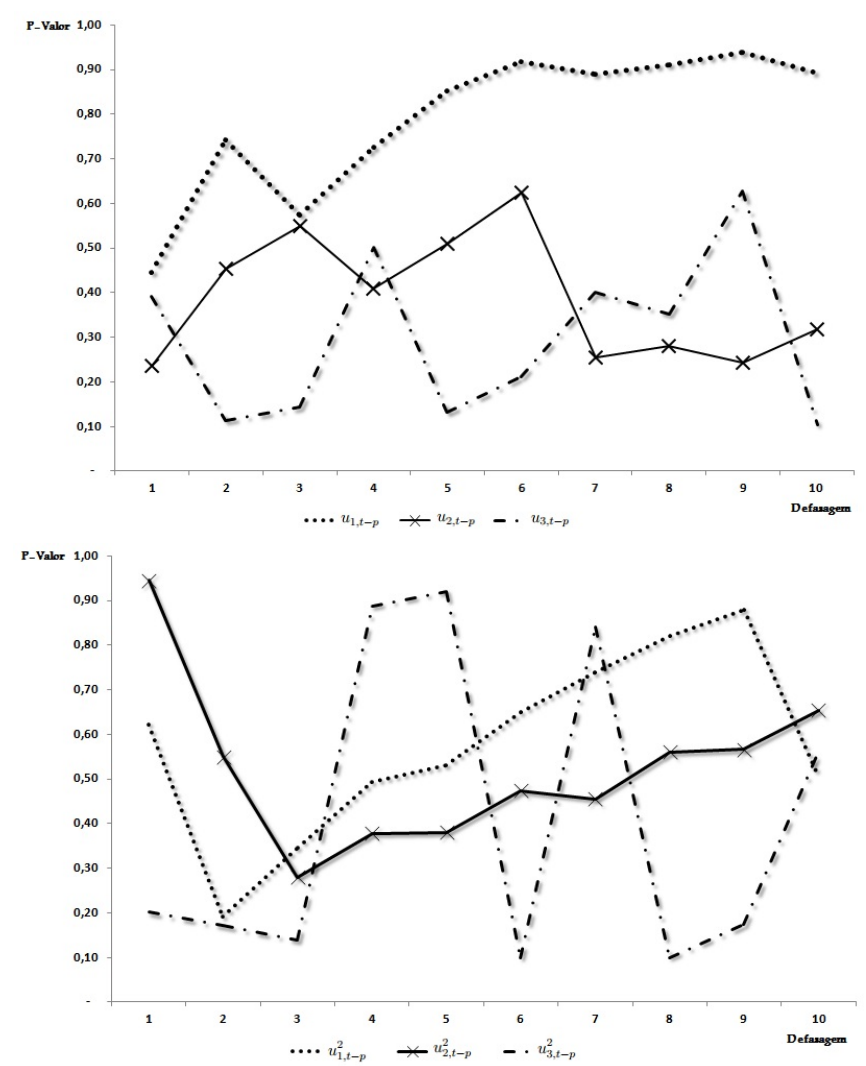

590 Rev. Bras. Finanças (Online), Rio de Janeiro, V15, No. 4, Oct. 201 
Figura 3

Teste LM para autocorrelação Para Resíduos e Quadrado dos Resíduos - Modelo 2

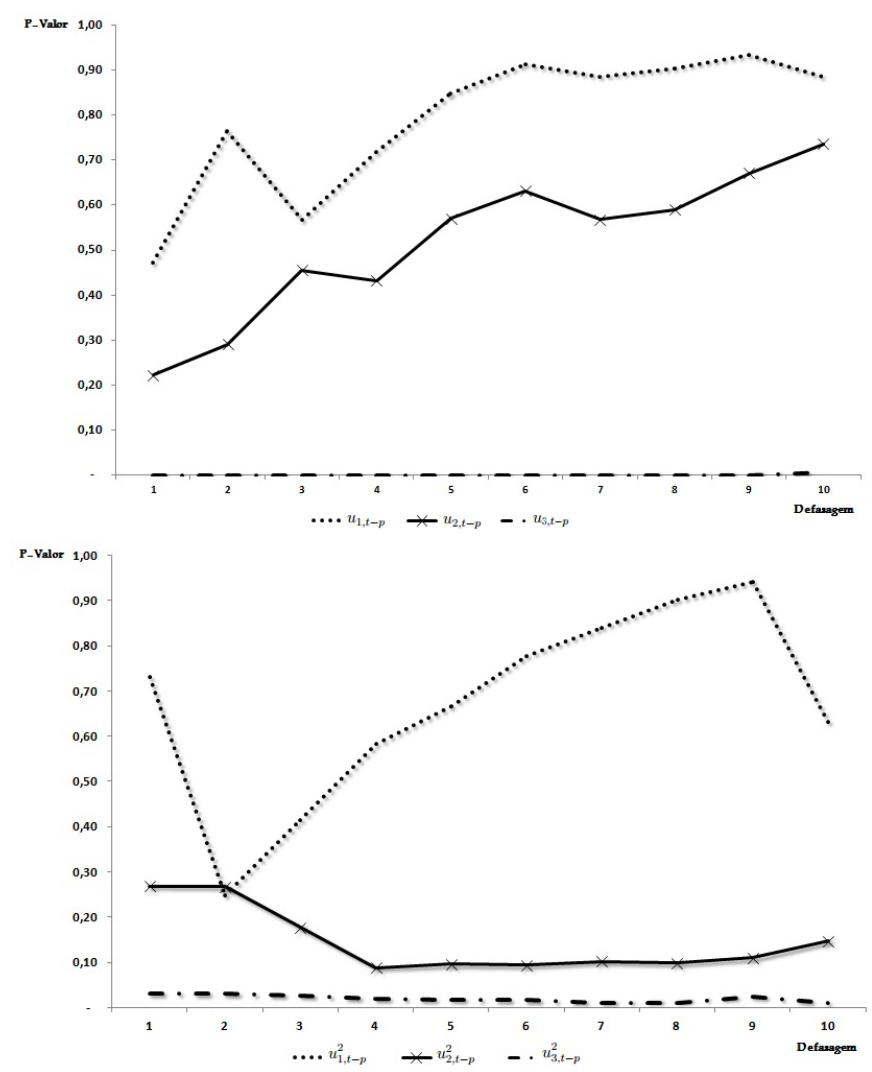

A partir dos modelos adequadamente estimados e validados, obtemos as correlações condicionais estimadas e parâmetros que serão utilizados nos testes de causalidade Granger de segunda ordem e nas análises comparativas que se seguirão.

\subsection{Testes de Causalidade de Granger de Segunda Ordem}

Os testes de causalidade de Granger de segunda ordem, são implementados a partir dos parâmetros estimados dos modelos apresentados, dessa forma apresentamos a seguir os resultados:

O primeiro modelo que utiliza uma proxy para choques cambiais com 48 taxas cambiais, ou seja um choque cambial mais amplo, teve comprovada a existência dos efeitos de spillover na direção da volatilidade dos choques, cambiais e de mercados financeiros internacionais,

(9) (1) Rev. Bras. Finanças (Online), Rio de Janeiro, V15, No. 4, Oct. 2017591 
Figura 4

Teste LM para autocorrelação Para Resíduos e Quadrado dos Resíduos - Modelo 3
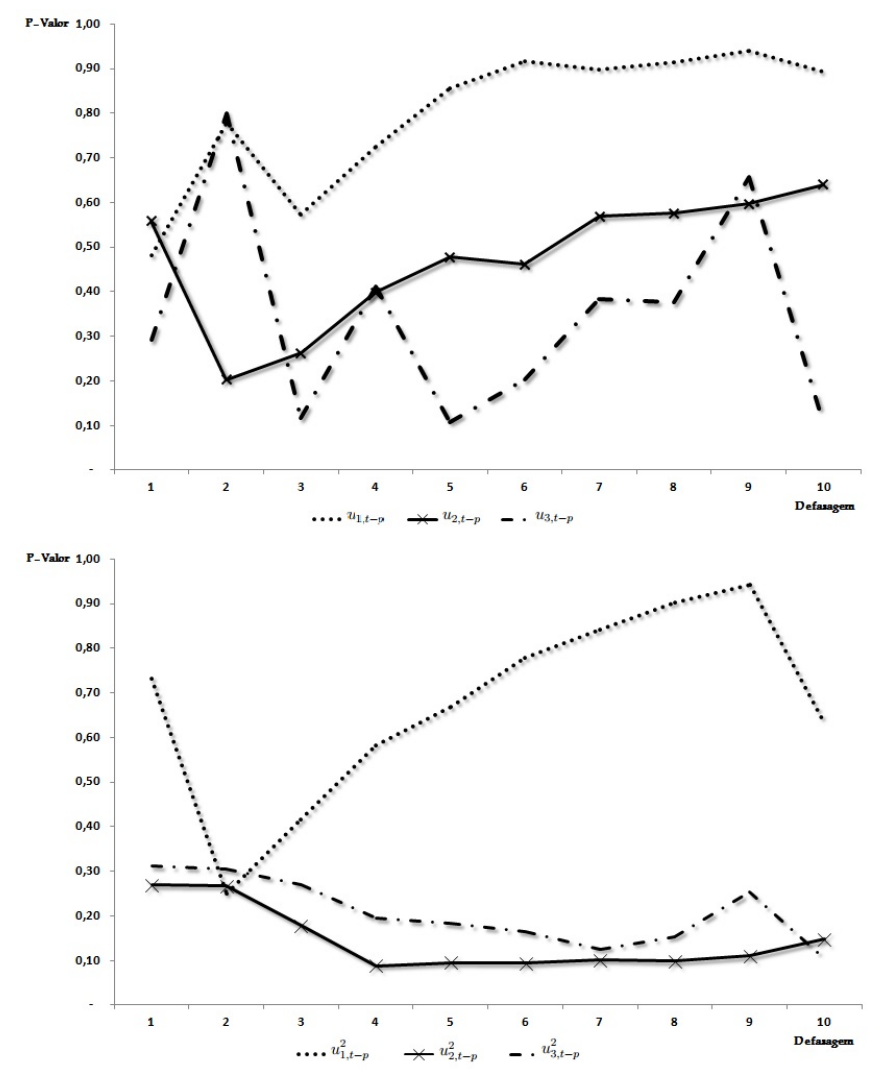

Tabela 5

Teste Causalidade de Granger de Segunda Ordem - Modelo 1

\begin{tabular}{|c|c|c|c|c|}
\hline \multicolumn{5}{|c|}{ Teste CG Segunda Ordem com 10 defasagens } \\
\hline & Ibovespa & IND1 & IND3 & Teste Conjunto \\
\hline Ibovespa & 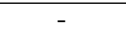 & 0,205 & 0,415 & 0,391 \\
\hline IND1 & 0,003 & - & 0,113 & 0,030 \\
\hline IND3 & 0,010 & 0,073 & - & 0,009 \\
\hline
\end{tabular}

para a volatidade do Ibovespa. O teste conjunto mostra que não há causalidade no sentido de Granger, da volatilidade do Ibovespa para a volatilidade dos choques definidos para esse modelo.

A exclusão do dólar na proxy do IND2, não altera os resultados

592 Rev. Bras. Finanças (Online), Rio de Janeiro, V15, No. 4, Oct. 201 
Tabela 6

Teste Causalidade de Granger de Segunda Ordem - Modelo 2

\begin{tabular}{|c|c|c|c|c|}
\hline \multicolumn{5}{|c|}{ Teste CG Segunda Ordem com 10 defasagens } \\
\hline & Ibovespa & IND1 & IND3 & Teste Conjunto \\
\hline Ibovespa & 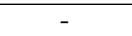 & 0,958 & 0,549 & 0,837 \\
\hline IND2 & 0,000 & - & 0,003 & 0,000 \\
\hline IND3 & 0,013 & 0,126 & - & 0,013 \\
\hline
\end{tabular}

de transbordamento de volatilidade, ou seja, existe causalidade no sentido de Granger da volatilidade cambial e de mercados financeiros internacionais para a volatilidade do Ibovespa.

Tabela 7

Teste Causalidade de Granger de Segunda Ordem - Modelo 3

\begin{tabular}{|c|c|c|c|c|}
\hline \multicolumn{5}{|c|}{ Teste CG Segunda Ordem com 10 defasagens } \\
\hline & Ibovespa & IND1 & IND3 & Teste Conjunto \\
\hline Ibovespa & - & 0,198 & 0,449 & 0,344 \\
\hline Dólar & 0,0567 & - & 0,117 & 0,0282 \\
\hline IND3 & 0,043 & 0,096 & - & 0,013 \\
\hline
\end{tabular}

Quando consideramos o dólar como proxy para um choque cambial, os resultados se mantem, ou seja, existe evidência estatísticamente significante de efeitos de spillover da volatilidade do dólar e do mercado financeiro internacional causando no sentido de Granger, a volatilidade do Ibovespa. Esses resultados apresentados estão em linha com os resultados obtidos pelos principais estudos internacionais, que identificaram a existência de transbordamento entre a volatilidade câmbial e a volatilidade de mercados financeiros.

\subsection{Análises Comparativas}

Nesse seção, com a evidência de existência de efeitos de spillovers observado sempre ocorrendo na direção dos choques para a volatilidade do Ibovespa, um conjunto de comparações estatística são implementadas com a finalidade de identificar qual transbordamento cambial, ou seja, qual correlação condicional estimada, em termos de magnitudo é mais representativo. Para tanto aplicamos testes de média ${ }^{4}$ para inferência dessas comparações.

\footnotetext{
${ }^{4}$ Testes $\mathrm{T}$ unicaudais com variâncias desiguais
} 
Figura 5

Correlações Condicionais de Choques Cambiais

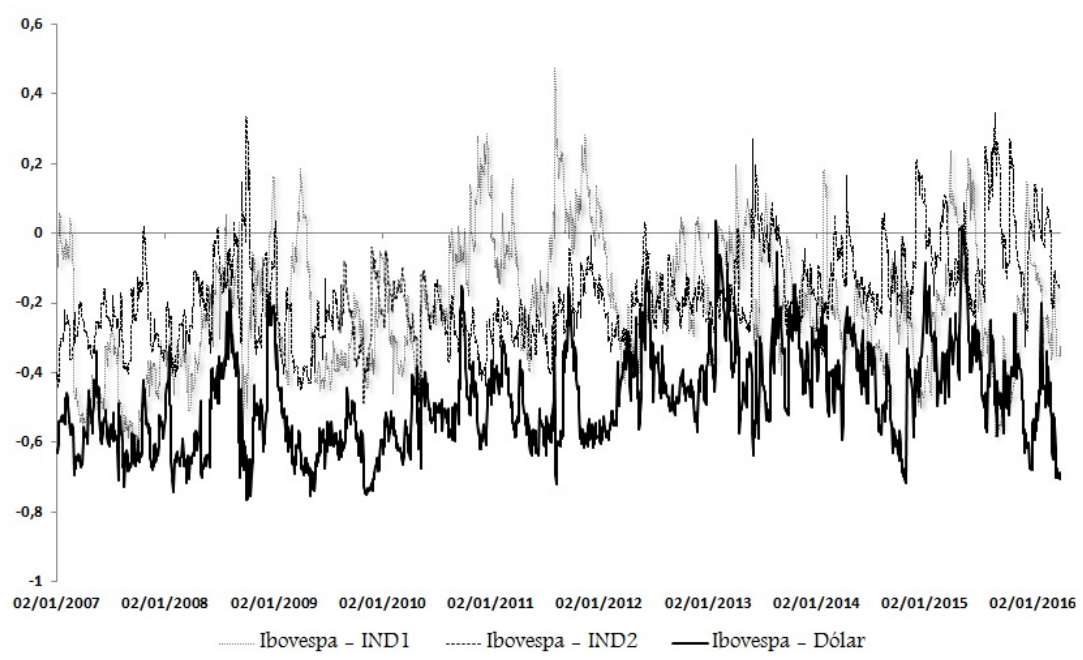

Tabela 8

Teste T para Comparações

\begin{tabular}{c|c}
\hline Resultado do Teste T & Correlações Condicionais \\
\hline 1 & Ibovespa, IND1 > Ibovespa, IND2 \\
0 & Ibovespa, IND1 $\leq$ Ibovespa, Dólar \\
0 & Ibovespa, IND2 $\leq$ Ibovespa, Dólar \\
\hline \multicolumn{2}{c}{$H_{0}: y(i) \leq y(j)$, com $i \neq j}$.
\end{tabular}

1:Rejeita-se $H_{0}$ ao nível de significância de $5 \%$.

Como o IND1 representa um conjunto de todos os câmbios considerados e IND2 exclui o dólar, foi possível entender a importância do dólar por meio desses dois controles. O gráfico acima evidencia, que o dólar apresenta valores de correlação condicional negativamente maiores. A evidência gráfica é corroborada pelos testes que indicam sempre uma maior correlação condicional associada à presença do dólar, sejam em conjunto com as demais taxas de cambio, seja isoladamente, com a maior correlação condicional negativa observada. Ou seja, quanto maior a volatilidade cambial menor volatilidade do mercado acionário brasileiro. Com a importância do dólar destacada, utilizamos o modelo 3 para analisarmos os efeitos de spillovers de mercados financeiros internacionais:

Os tranbordamentos de volatilidade de mercados financeiros in-

594 Rev. Bras. Finanças (Online), Rio de Janeiro, V15, No. 4, Oct. 201 


\section{Figura 6}

Correlações Condicionais, Volatilidade e Índice Ibovespa

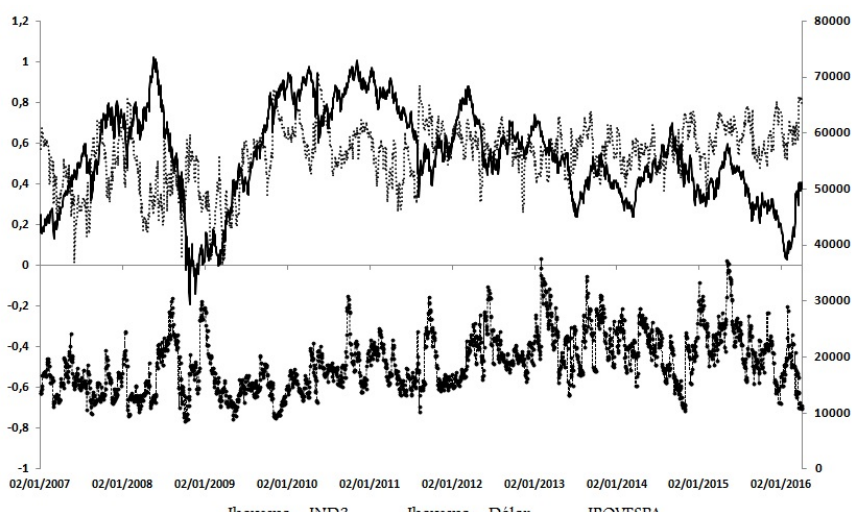

...... Ibovespa - IND3 …. lbovespa - Dólar —IBOVESPA

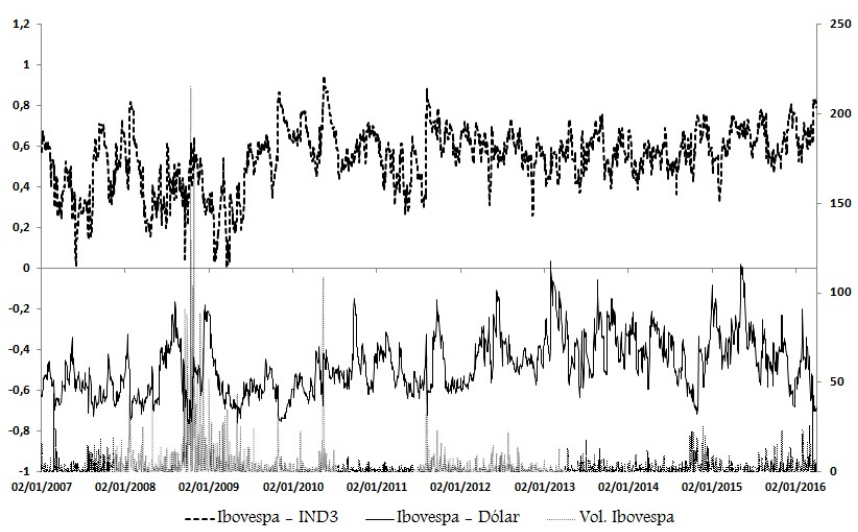

ternacionais apresentam correlações condicionais positivas, ou seja, aumento de volatilidade dos mercados financeiros internacionais está associado a um aumento da volatilidade do mercado acionário brasileiro. Outro ponto de destaque está relacionado a evidência gráfica de que em períodos de crise, como a crise de 2008, as correlações condicionais tem uma menor magnitude, contudo essa evidência carece de um estudo de evento para sua comprovação.

\section{Conclusões}

O presente estudo se propôs em sua primeira parte construir um referencial teórico da literatura que tratar dos efeitos de spillover em suas diferentes abordagens, transbordamentos de câmbios e de 
mercados financeiros internacionais.

O estudo verificou a possibilidade de construção de proxies para choques cambiais e de mercados financeiros internacionais a partir do uso de ACP, de forma que tais índices (ou retornos representativos) construídos apresentassem dinâmica temporal consistente com os fatos estilizados de retornos, o que possibilitou seu uso para estimar os efeitos de transbordamento de cada tipo (Cambial e de mercados financeiros internacionais).

As estimações de modelos MGARCH BEKK que eliminaram autocorrelação dos resíduos padronizados e de seus quadrados indicam que tais modelos foram capazes de produzir uma boa representação dos processos geradores das séries. Esses modelos produziram correlações condicionais, as quais com dinâmica temporal permitiram concluir que os transbordamentos não são eventos estáticos. Os testes de causalidade de Granger (teste $\mathrm{HH}$ ) de segunda ordem não só validaram a existência de spillover (Cambiais e de mercados financeiros internacionais) quanto sua direção. Os resultados mostram que a causalidade no sentido de Granger sempre ocorre dos choques para o índice Ibovespa, ou seja, existe uma precedência estatisticamente significante entre transbordamentos cambiais e de mercados financeiros internacionais e o índice Ibovespa.

As correlações condicionais estimadas apresentam valores positivos quando considerado a Proxy para mercados financeiros internacionais e negativos para Proxy de choques cambiais (ou mesmo do Dólar). Ou seja, esses resultados indicam que aumentos da volatilidade cambial estão associados a uma redução da volatilidade do índice Ibovespa e aumentos da volatilidade de mercados financeiros internacionais estão associados a aumentos da volatilidades do índice Ibovespa.

Os testes comparando essas correlações condicionais estimadas permitiram evidenciar a importância da volatilidade do Dólar em termos de transbordamento com o uso das outras proxies de choques cambias com diferentes combinações de moedas. Todas essas conclusões precisam considerar questões não abordadas nesse estudo, para que sejam definitivas. Outros modelos GARCH Multivariados (DVEC, DCC, CCC, etc) devem ser testados e seus resultados de correlações condicionais comparados afim de avaliar a consistência desses valores entre diferentes modelos. não foi considerado nesse 
estudo a possibilidade de memória longa nas séries do Bovespa e nas series geradas via $\mathrm{ACP}$, também não foram realizados testes de quebra estruturais tanto para as séries quanto para os testes de causalidade de Granger de segunda ordem conforme apresentado em van Dijk et al. (2005). Outra possibilidade não considerada foram os modelos MGARCH Cópulas, resgardados contudo pelo fato de não haver testes de causalidade de Granger de segunda ordem de modelo MGARCH Cópulas o que direcionou o estudo para o modelos MGARCH com possibilidade de aplicação direta dos testes, além de outros aspectos.

Outras extensões podem diretamente seguir esse estudo, incorporando outros tipos de choques, como por exemplo, choques de commodities, de risco país etc. Outra extensão natural seria a utilização dos modelos MGARCH Fatoriais com versões que já possibilitam tratar questões de memória longa conforme apresentado em Alessi et al. (2007). Não obstante as questões salientadas, concluímos o estudo ressaltando seu caráter pioneiro para a investigação de transbordamentos de dois diferentes tipos de choques estimados em um mesmo modelo, e em particular para o caso brasileiro.

\section{Referências}

Ajayi, R. A. and Mougoue, M. (1996). On the dynamic relation between stock prices and exchange rates. Journal of Financial Research, 19(2):193-207.

Alaganar, V. and Bhar, R. (2007). Empirical properties of currency risk in country index portfolios. The Quarterly Review of Economics and Finance, 47(1):159-174.

Alessi, L., Barigozzi, M., Capasso, M., et al. (2007). Dynamic factor garch: multivariate volatility forecast for a large number of series. Laboratory of Economics and Management (LEM), SantVAnna School of Advanced Studies.

Amihud, Y. (1994). Exchange rates and the valuation of equity shares. Exchange Rates and Corporate Performance, 11:49-59.

Bae, K.-H. and Karolyi, G. A. (1994). Good news, bad news and international spillovers of stock return volatility between japan and the us. Pacific-Basin Finance Journal, 2(4):405-438.

(9) (๑) Rev. Bras. Finanças (Online), Rio de Janeiro, V15, No. 4, Oct. 2017597 
Baele, L. (2005). Volatility spillover effects in european equity markets. Journal of Financial and Quantitative Analysis, 40(02):373401.

Bartov, E. and Bodnar, G. M. (1994). Firm valuation, earnings expectations, and the exchange-rate exposure effect. The Journal of Finance, 49(5):1755-1785.

Black, F. (1976). Studies of stock price volatility changes, proceedings of the 1976 meetings of the business and economic statistics section. 177-191. In American Statistical association.

Bollerslev (1986). Generalized autoregressive conditional heteroskedasticity. Journal of Econometrics, 31(3):307-327.

Bollerslev, T., Engle, R. F., and Wooldridge, J. M. (1988). A capital asset pricing model with time-varying covariances. The Journal of Political Economy, pages 116-131.

Booth, L. and Rotenberg, W. (1990). Assessing foreign exchange exposure: Theory and application using canadian firms*. Journal of International Financial Management $\&$ Accounting, 2(1):1-22.

Branson, W. H. (1983). A model of exchange-rate determination with policy reaction: evidence from monthly data.

Caldeira, A. M., Souza, R. C., and Machado, M. A. S. (2010). Identificação automática das ordens dos modelos garch utilizando redes neurais. ENGEVISTA, 11(2).

Carruth, A., Dickerson, A., and Henley, A. (2000). What do we know about investment under uncertainty? Journal of Economic Surveys, 14(2):119-154.

Chen, J., Naylor, M., and Lu, X. (2004). Some insights into the foreign exchange pricing puzzle: Evidence from a small open economy. Pacific-Basin Finance Journal, 12(1):41-64.

Cheung, Y.-W. and Ng, L. K. (1996). A causality-in-variance test and its application to financial market prices. Journal of Econometrics, $72(1): 33-48$.

598 Rev. Bras. Finanças (Online), Rio de Janeiro, V15, No. 4, Oct. 201 
Chkili, W. and Nguyen, D. K. (2014). Exchange rate movements and stock market returns in a regime-switching environment: Evidence for brics countries. Research in International Business and Finance, 31:46-56.

Christie, A. A. (1982). The stochastic behavior of common stock variances: Value, leverage and interest rate effects. Journal of Financial Economics, 10(4):407-432.

Comte, F. and Lieberman, O. (2003). Asymptotic theory for multivariate garch processes. Journal of Multivariate Analysis, 84(1):6184 .

Darby, J., Hallett, A. H., Ireland, J., and Piscitelli, L. (1999). The impact of exchange rate uncertainty on the level of investment. The Economic Journal, 109(454):55-67.

Diamandis, P. F. and Drakos, A. A. (2011). Financial liberalization, exchange rates and stock prices: Exogenous shocks in four latin america countries. Journal of Policy Modeling, 33(3):381-394.

DiCesare, G. (2006). Imputation, estimation and missing data in finance. $\mathrm{PhD}$ thesis.

Diebold, F. X. and Yilmaz, K. (2009). Measuring financial asset return and volatility spillovers, with application to global equity markets*. The Economic Journal, 119(534):158-171.

Diebold, F. X. and Yilmaz, K. (2015). Trans-atlantic equity volatility connectedness: Us and european financial institutions, 2004-2014. Journal of Financial Econometrics, 14(1):81-127.

Dornbusch, R. and Fischer, S. (1980). Exchange rates and the current account. The American Economic Review, 70(5):960-971.

Engle, R. F. (1982). Autoregressive conditional heteroscedasticity with estimates of the variance of united kingdom inflation. Econometrica, pages $987-1007$.

Fedorova, E. and Saleem, K. (2009). Volatility spillovers between stock and currency markets: Evidence from emerging eastern europe.

(9) (1) Rev. Bras. Finanças (Online), Rio de Janeiro, V15, No. 4, Oct. 2017599 
Frankel, J. A. and Froot, K. A. (1985). Using survey data to test some standard propositions regarding exchange rate expectations.

Franses, P. H. and Van Dijk, D. (2000). Non-linear Time Series Models in Empirical Finance. Cambridge University Press.

Glosten, L. R., Jagannathan, R., and Runkle, D. E. (1993). On the relation between the expected value and the volatility of the nominal excess return on stocks. The Journal of Finance, 48(5):1779-1801.

Goldberg, L. S. (1993). Exchange rates and investment in united states industry. The Review of Economics and Statistics, pages $575-588$.

Hafner, C. M. and Herwartz, H. (1998). Structural analysis of portfolio risk using beta impulse response functions. Statistica Neerlandica, 52(3):336-355.

Hafner, C. M. and Herwartz, H. (2008). Testing for causality in variance using multivariate garch models. Annales d'Economie et de Statistique, pages 215-241.

Hamao, Y., Masulis, R. W., and Ng, V. (1990). Correlations in price changes and volatility across international stock markets. Review of Financial studies, 3(2):281-307.

Herwartz, H. and Lütkepohl, H. (2000). Multivariate volatility analysis of vw stock prices. International Journal of Intelligent Systems in Accounting, Finance 83 Management, 9(1):35-54.

Hoti, S., Chan, F., McAleer, M., et al. (2002). Structure and asymptotic theory for multivariate asymmetric volatility: Empirical evidence for country risk ratings. In Australasian Meeting of the Econometric Society, Brisbane, Australia, page 2002.

IMF (2016). Global financial stability report fostering stability in a low-growth, low-rate era. Technical report, International Monetary Fund.

Jorion, P. (1990). The exchange-rate exposure of us multinationals. Journal of Business, pages 331-345.

600 Rev. Bras. Finanças (Online), Rio de Janeiro, V15, No. 4, Oct. 201 
Kanas, A. (1998). Volatility spillovers across equity markets: European evidence. Applied Financial Economics, 8(3):245-256.

Kanas, A. (2000). Volatility spillovers between stock returns and exchange rate changes: International evidence. Journal of Business Finance \&s Accounting, 27(3-4):447-467.

Lütkepohl, H. (1993). Testing for causation between two variables in higher-dimensional var models. In Studies in Applied Econometrics, pages $75-91$. Springer.

Lütkepohl, H. (2005). New introduction to multiple time series analysis. Springer Science \& Business Media.

Miyakoshi, T. (2003). Spillovers of stock return volatility to asian equity markets from japan and the us. Journal of International Financial Markets, Institutions and Money, 13(4):383-399.

Morales, L. d. l. N. (2008). Volatility spillovers between equity and currency markets: Eviderice from major latin american countries. Cuadernos de Economía, 45(132):185-215.

Muradoglu, G., Taskin, F., and Bigan, I. (2000). Causality between stock returns and macroeconomic variables in emerging markets. Russian 8 East European Finance and Trade, 36(6):33-53.

Phylaktis, K. and Ravazzolo, F. (2005). Stock prices and exchange rate dynamics. Journal of International Money and Finance, 24(7):1031-1053.

Susmel, R. and Engle, R. F. (1994). Hourly volatility spillovers between international equity markets. Journal of International Money and Finance, 13(1):3-25.

Theodossiou, P. and Lee, U. (1993). Mean and volatility spillovers across major national stock markets: Further empirical evidence. Journal of Financial Research, 16(4):337-350.

van Dijk, D., Osborn, D. R., and Sensier, M. (2005). Testing for causality in variance in the presence of breaks. Economics Letters, 89(2):193-199.

(9) (1) Rev. Bras. Finanças (Online), Rio de Janeiro, V15, No. 4, Oct. 2017601 
Veiga, B. and McAleer, M. (2003). Multivariate volatility and spillover effects in financial markets. Complexity and Integrated Resources Management, page 1442.

Wei, K. J., Liu, Y.-J., Yang, C.-C., and Chaung, G.-S. (1995). Volatility and price change spillover effects across the developed and emerging markets. Pacific-Basin Finance Journal, 3(1):113-136.

Yang, S.-Y. and Doong, S.-C. (2004). Price and volatility spillovers between stock prices and exchange rates: empirical evidence from the g-7 countries. International Journal of Business and Economics, 3(2):139.

Zapatero, F. (1995). Equilibrium asset prices and exchange rates. Journal of Economic Dynamics and Control, 19(4):787-811.

\section{Apêndice}

Tabela 9

Taxas Cambiais Utilizadas

\begin{tabular}{|c|c|c|c|c|c|}
\hline & País & Moeda & & País & Moeda \\
\hline 1 & Alemanha & Marco/US\$ Irlanda & 25 & Irlanda & Libra Irlandesa (Punts)/US\$ \\
\hline 2 & Argentina & Peso Argentino/US\$ & 26 & Israel & Shekel/US\$ \\
\hline 3 & Austrália & Dólar Australiano/US\$ & 27 & Itália & Lira/US\$ \\
\hline 4 & Áustria & Schilling/US\$ & 28 & Japão & Yen/US\$ \\
\hline 5 & Bolívia & Boliviano/US\$ & 29 & Coreia & Won/US\$ \\
\hline 6 & Brasil & Real /US\$ & 30 & Malásia & Ringgit/US\$ \\
\hline 7 & Bulgária & Lev/US\$ & 31 & México & Peso Mexicano/US\$ \\
\hline 8 & Canadá & Dólar Canadense/US\$ & 32 & Noruega & Coroa Norueguesa/US\$ \\
\hline 9 & Chile & Peso Chileno/US\$ & 33 & Nova Zelândia & Dólar da Nova Zelândia/US\$ \\
\hline 10 & China & Yuan/US\$ & 34 & Paraguai & Guarani/US\$ \\
\hline 11 & Cingapura & Dólar de Singapura/US\$ & 35 & Peru & Novo Sol do Peru/US\$ \\
\hline 12 & Colômbia & Peso Colombiano/US\$ & 36 & Polônia & Zloty da Polônia/US\$ \\
\hline 13 & Equador & Sucre/US\$ & 37 & Romênia & Leu/US\$ \\
\hline 14 & Eslováquia & Coroa Eslovaca/US\$ & 38 & Rússia & Rublo/US\$ \\
\hline 15 & Eslovênia & Dólar da Eslovênia/US\$ & 39 & Suécia & Coroa Sueca/US\$ \\
\hline 16 & Espanha & Peseta/US\$ & 40 & Suíça & US\$/Franco Suiço \\
\hline 17 & Euro & US\$/Euro & 41 & Tailândia & Bath/US\$ \\
\hline 18 & Filipinas & Peso Filipino/US\$ & 42 & Taiwan & Dólar de Taiwan/US\$ \\
\hline 19 & França & Franco/US\$ & 43 & Tcheca & Coroa Tcheca/US\$ \\
\hline 20 & Hong Kong & Dólar de Hong Kong/US\$ & 44 & Turquia & Nova Lira da Turquia/US\$ \\
\hline 21 & Hungria & Forint/US\$ & 45 & Ucrânia & Hyvnia da Ucrânia/US\$ \\
\hline 22 & Índia & Rúpia da Índia/US\$ & 46 & Uruguai & Peso do Uruguai/US\$ \\
\hline 23 & Indonésia & Rúpia da Indonésia/US\$ & 47 & África do Sul & US\$/ZAR \\
\hline 24 & Inglaterra & US\$/Libra & 48 & Vietnã & Dong/US\$ \\
\hline
\end{tabular}

602 Rev. Bras. Finanças (Online), Rio de Janeiro, V15, No. 4, Oct. 201 
Spillovers de Volatilidades Cambiais e de Mercados Financeiros Internacionais no Mercado Acionário Brasileiro

Tabela 10

Bolsas de valores utilizadas

\begin{tabular}{ccc}
\hline & País & Índice \\
\hline 1 & Alemanha & DAX \\
2 & Argentina & Merval \\
3 & Austrália & ASX \\
4 & Chile & Chile Stock Mkt \\
5 & Colômbia & IGBC General \\
6 & França & CAC 40 \\
7 & Hong Kong & Hang Seng \\
8 & Londres & FTSE 100 \\
9 & México & Bolsa do México \\
10 & Nasdaq & Nasdaq \\
11 & Nova York & Dow Jones \\
12 & Peru & Lima General Index \\
13 & S\&P 500 & S\&P 500 \\
14 & Japão & Nikkei 225 \\
15 & Venezuela & Venezuela Stock mkt \\
16 & Xangai & Shanghai SE \\
17 & Europa & EU Stoxx \\
\hline
\end{tabular}

@. (-) Rev. Bras. Finanças (Online), Rio de Janeiro, V15, No. 4, Oct. 2017603 